\title{
Optical and system performance of SPEXone, a multi-angle channeled spectropolarimeter for the NASA PACE mission
}

Jeroen H. Rietjens, Jochen Campo, Martijn Smit, Robbert Winkelman, Raj Nalla, et al.

Jeroen H. H. Rietjens, Jochen Campo, Martijn Smit, Robbert Winkelman, Raj Nalla, Jochen Landgraf, Otto Hasekamp, Marc Oort, Aaldert van Amerongen, "Optical and system performance of SPEXone, a multi-angle channeled spectropolarimeter for the NASA PACE mission," Proc. SPIE 11852, International Conference on Space Optics - ICSO 2020, 1185234 (11 June 2021); doi: 10.1117/12.2599531

SPIE Event: International Conference on Space Optics - ICSO 2021, 2021, Online Only 


\section{International Conference on Space Optics-ICSO 2020}

Virtual Conference

30 March-2 April 2021

Edited by Bruno Cugny, Zoran Sodnik, and Nikos Karafolas
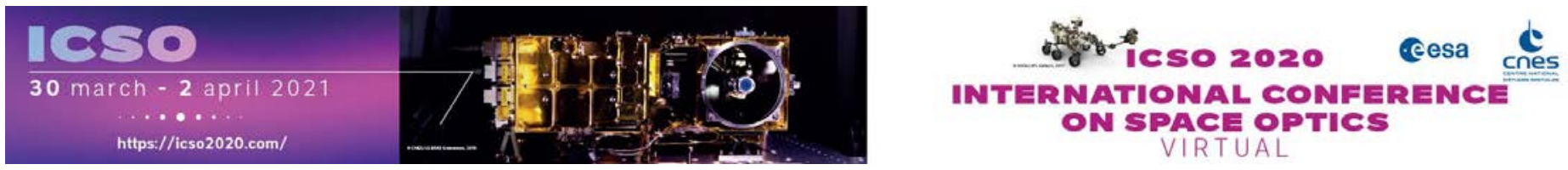

\section{Optical and system performance of SPEXone, a multi-angle channeled spectropolarimeter for the NASA PACE mission}

\section{Cesa isoporecestings denes}




\title{
Optical and system performance of SPEXone, a multi-angle channeled spectropolarimeter for the NASA PACE mission
}

\author{
Jeroen Rietjens ${ }^{\mathrm{a}}$, Jochen Campo ${ }^{\mathrm{a}}$, Martijn Smit ${ }^{\mathrm{a}}$, Robbert Winkelman ${ }^{\mathrm{b}}$, Raj Nalla ${ }^{\mathrm{b}}$, Jochen \\ Landgraf $^{\mathrm{a}}$, Otto Hasekamp ${ }^{\mathrm{a}}$, Marc Oort ${ }^{\mathrm{b}}$, Aaldert van Amerongen ${ }^{\mathrm{a}}$, and SPEXone team ${ }^{\mathrm{a}, \mathrm{b}}$

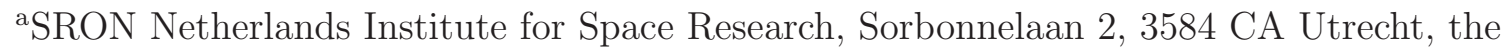 \\ Netherlands \\ ${ }^{\mathrm{b}}$ Airbus Defence and Space Netherlands, Mendelweg 30, 2333 CS Leiden, the Netherlands
}

\begin{abstract}
SPEXone is a multi-angle channeled spectropolarimeter that is developed by a Dutch consortium consisting of SRON and Airbus Defence and Space Netherlands with support from TNO. SPEXone will fly together with the Ocean Color Instrument (OCI) and the Hyper-Angular Rainbow Polarimeter-2 (HARP-2) on the NASA Plankton, Aerosol, Clouds and ocean Ecosystem (PACE) mission, which has a notional launch in 2023. SPEXone will deliver high quality hyperspectral multi-angle radiance and polarization products that, together with products from OCI and HARP2, enable unprecedented aerosol and cloud characterization from space.
\end{abstract}

SPEXone employs dual beam spectral polarization modulation, in which the state of linear polarization is encoded in a spectrum as a periodic variation of the intensity. This technique enables high polarimetric accuracies in operational environments, since it provides snapshot acquisition of both radiance and polarization without moving parts. SPEXone has five viewing angles that are realized using a novel three-mirror segmented telescope assembly. The telescope focuses light captured by the five viewing angles onto a single image plane consisting of five stacked sub-slits. This multi-slit forms the entrance slit of a reflective grating spectrometer that consists of freeform mirrors and an order-sorting filter close to the focal plane, yielding an intrinsic spectral resolution of 2 $\mathrm{nm}$ and $5.4 \mathrm{~km}$ spatial resolution across the $100 \mathrm{~km}$ swath. The spectrometer re-images two spectral images per viewing angle following a dual beam spectral polarization modulation implementation.

In this contribution, the optical performance of the telescope and spectrometer will be presented by means of star stimulus measurements at the slit plane and at the spectrometer focal plane. Measurements of the optical spot quality and preliminary measurements of stray light are compared with the optical design and with stray light simulations. We find that the measured optical performance of the telescope and spectrometer is better than modelled, showing higher resolution and lower slit keystone, thereby meeting all spatial and spectral resolution requirements. Also, preliminary stray light results indicate a higher diffuse but lower ghost contribution to the total stray light, which is in general beneficial for implementing stray light correction, which will enhance the polarimetric accuracy in inhomogeneous scenes.

Keywords: spectropolarimetry, spectral modulation, multi-angle, remote sensing, atmospheric aerosol, SPEXone, PACE

\section{INTRODUCTION}

SPEXone ${ }^{1}$ is a compact multi-angle spectropolarimeter developed by a partnership of SRON Netherlands Institute for Space Research and Airbus Defence and Space Netherlands with support from TNO. SPEXone is a contributed payload to the NASA Plankton, Aerosol, Cloud and ocean Ecosystem (PACE ${ }^{2}$ ) mission, which is developed by NASA Goddard Space Flight Center (GSFC). PACE has a notional launch in 2023 and a minimum mission duration of three years, with orbit maintenance capabilities for 10 years. The PACE mission will make global observations of the atmosphere and ocean surface, with the goal to understand and quantify the global biogeochemical cycling and ecosystem function in response to anthropogenic and natural environmental variability and change. Besides SPEXone, the PACE observatory includes two other instruments: the primary

Corresponding author: Jeroen Rietjens, e-mail: j.h.h.rietjens@sron.nl 
Ocean Color Instrument (OCI), and a second supporting polarimeter, HARP-2. ${ }^{3}$ SPEXone will deliver high quality hyperspectral multi-angle radiance and polarization products that, together with products from OCI and HARP2, enable unprecedented aerosol and cloud characterization from space.

SPEXone builds on heritage of a SPEX-family of instruments. Handheld (iSPEX ${ }^{4}$ ), ground-based (ground$\mathrm{SPEX}^{5}$ ) and airborne (SPEX airborne ${ }^{6,7}$ ) versions have shown the capability of yielding high quality polarimetric data that enables the retrieval of aerosol properties. ${ }^{8,9}$ SPEX airborne data obtained during the ACEPOL campaign using NASA's ER-2 airplane has demonstrated an agreement with benchmark polarimeters of about 0.005 . For SPEXone on the NASA PACE mission, and for other future space-borne multi-angle polarimeters, an even higher polarimetric accuracy of 0.003 is required. ${ }^{10,11}$

This paper presents and discusses the optical performance of telescope and spectrometer of the SPEXone instrument. We focus on two aspects: the optical quality at the telescope and spectrometer focal planes by comparing the as-designed performance with measurements of the as-build instrument, and simulations and preliminary measurements of stray light. The former will be related to the overall system performance in terms of spatial and spectral resolution, and the latter will be discussed in relation to the expected polarimetric performance of the instrument in inhomogeneous scenes.

\section{SPEXONE INSTRUMENT CONCEPT}

SPEXone is a compact spectropolarimeter that fits within an envelope of $150 \mathrm{~mm}$ x $290 \mathrm{~mm} \times 370 \mathrm{~mm}$. It uses spectral polarization modulation ${ }^{12}$ to accurately measure the state of linear polarization of the incident light in the spectral range from 385 to $770 \mathrm{~nm}$. Spectral polarization modulation is a polarimetry technique that enables snapshot spectropolarimetry with high accuracy without moving parts. An achromatic quarter-wave retarder with its fast axis oriented at $45^{\circ}$ with respect to the fast axis of a compound athermal multiple-order retarder results in a spectral modulation of the polarisation state of the incident light. A polarizing beam splitter transforms the polarisation modulation into an intensity modulation in two complementary optical beams that can be measured with a detector at the focal plane of a spectrometer. This dual beam implementation does not only lead to low sensitivity to systematic effects, it also allows the reconstruction of the absolute radiance spectrum at the spectral resolution of the spectrometer.

The common swath of each of the five viewing angles of SPEXone is about $100 \mathrm{~km}$, which is measured with a spatial sampling distance of $2.7 \mathrm{~km}$. SPEXone acquires push-broom spectral image data at a rate of $3 \mathrm{~Hz}$. With a satellite ground speed of $6.8 \mathrm{~km} / \mathrm{s}$ this corresponds to an along track sampling distance of $2.3 \mathrm{~km}$. The optical quality at the telescope focal plane (the entrance slit of the spectrometer) and in the spectrometer, in combination with motion smear during the exposure time of a single image, is aimed to achieve Nyquist sampling of a ground scene with a spatial resolution of 4.6 x $5.4 \mathrm{~km}$ (along track (ALT) x across track (ACT)).

At the spectrometer focal plane, SPEXone uses a compact camera module from 3Dplus that is equipped with a CMOSIS CMV4000 CMOS image sensor, a Field-Programmable Gate-Array (FPGA), SDRAM and flash memory. ${ }^{13}$ Raw $2 \mathrm{k}$ x 2k images are acquired by the CMOS detector at a rate of $15 \mathrm{~Hz}$. 5 subsequent images are co-added and binned in order to reduce the data volume of a single final image to $\approx 200$ kpixel. Image acquisition, binning and co-adding operations are all executed within the DEM by the FPGA using dedicated firmware.

The camera module is embedded in the detector module (DEM) that is controlled at a constant temperature within $\pm 0.15 \mathrm{~K}$. A radiator provides sufficient cooling power to the DEM and also serves as a heat sink for the opto-mechanics, which allows controlling the temperature of the optics within $\pm 1 \mathrm{~K}$ while operating at room temperature. ${ }^{14}$ Two (redundant) LEDs can provide a smooth illumination of the focal plane for detector monitoring at the native pixel level during the eclipse.

The Instrument Control Unit (ICU) is a separate unit that powers and commands the DEM, controls internal calibration LEDs, performs thermal control of the DEM and instrument opto-mechanics, handles the science and housekeeping data acquisition and communicates with the satellite. 

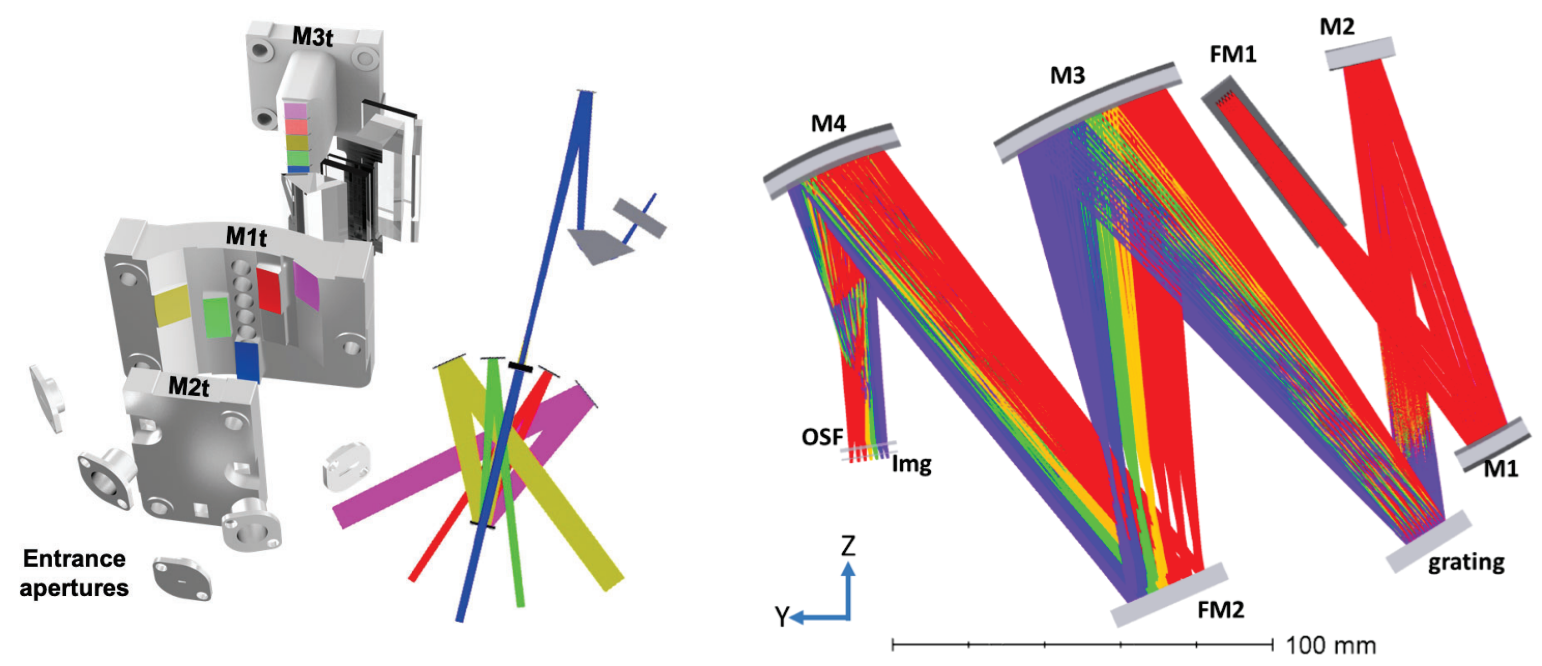

Figure 1. Left) Optical layout of SPEXone telescope in top and side view. Right) Spectrometer optical design.

\section{OPTICAL DESIGN}

The optical design of SPEXone consists of a reflective segmented telescope, transmissive polarization modulation optics (PMO), and a reflective grating spectrometer. The telescope is placed in front of a single PMO unit, which is advantageous since this subsystem yields the same response for all viewing angles. This approach also enables a compact design that can be well aligned with reduced integrating activities compared to the case of one PMO per viewing angle. The telescope consists of three segmented mirrors, each fabricated out of a single monolithic aluminium block, that capture the light from five viewing angles and project the individual swaths of each viewing angle onto a common slit plate, see Fig. 1. All five sub-mirrors of the three segmented mirrors are toroidal and together yield a telecentric beam towards the PMO.

The PMO comprising the optical elements for spectral polarization modulation accepts the beams from all five viewing angles, as described in. ${ }^{15} \mathrm{~A}$ polarizing beam splitter produces two complementary optical beams, which are recombined by two fold mirrors and a roof mirror, effectively doubling the slit height for the spectrometer and ensuring a symmetric ordering of the viewing angles with respect to the focal plane.

The spectrometer is based on a collimator consisting of two freeform mirrors, a reflection grating operating in first order, and an imager based on two freeform mirrors, see Fig. 1. One additional fold mirror in the imager is used to limit the volume of the spectrometer. The freeform mirrors in the collimator and imager are mainly utilised for correcting aberrations. The grating is a holographic reflection grating with 500 lines per $\mathrm{mm}$ operating in first order. To avoid order overlap at the focal plane, an order sorting filter is placed close to the detector. The order sorting filter is a fused silica substrate with a gradient filter that provides OD2.8 blocking of second order light on one side, and on the other side a short pass filter with a $50 \%$ cut-off at $800 \mathrm{~nm}$. All spectrometer components can be mounted from the outside into a common monolithic aluminium housing.

\section{OPTICAL PERFORMANCE}

The optical performance of SPEXone can be divided into three categories yielding different requirements for the optical quality of individual components:

1. Optical quality for ALT imaging: ALT imaging occurs at the entrance slit of the spectrometer and is therefore determined by the optical spot size produced by the telescope mirrors. In order to have a good definition of the instantaneous field of view, the spot size must be much smaller than the width of the entrance slit of $200 \mu \mathrm{m}$. The nominal as designed RMS full width spot sizes are smaller than $9 \mu \mathrm{m}$ for all viewing angles, leaving sufficient margin for alignment tolerancing and manufacturing specifications. 

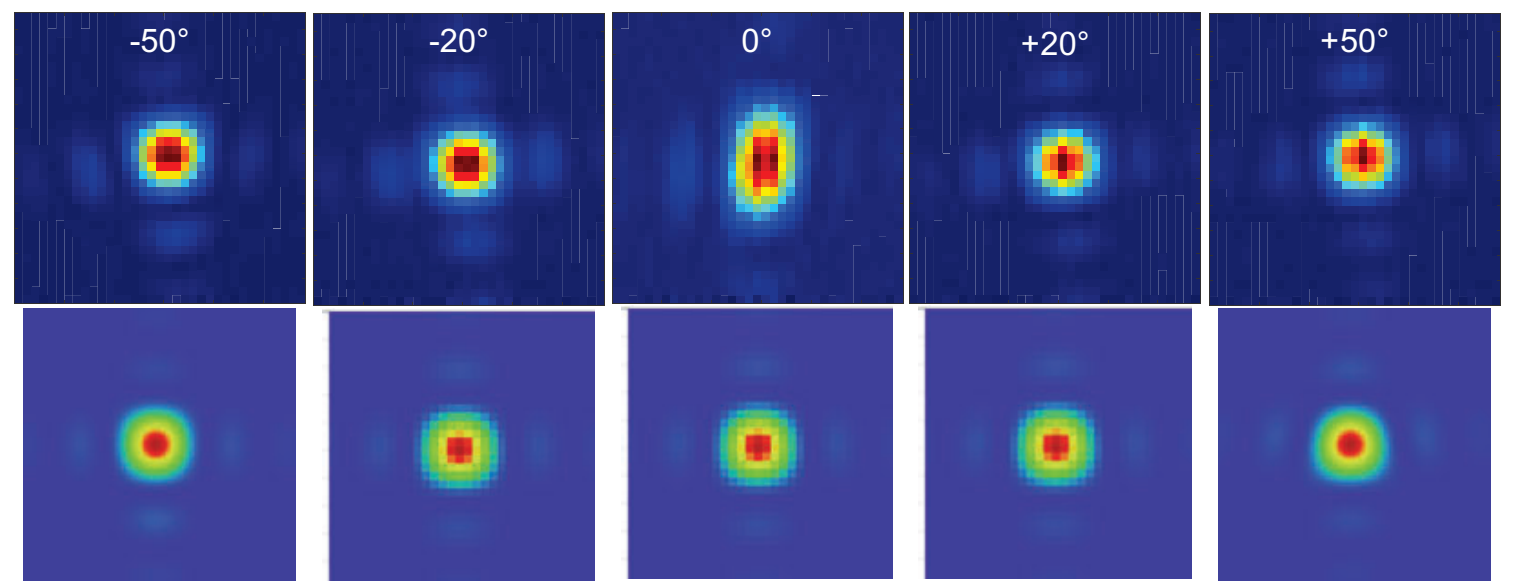

$50 \mu \mathrm{m}$

Figure 2. Spot images at the telescope focal plane for each of the five viewing angles under illumination by a star stimulus. Top: measured, bottom: simulated.

2. Optical quality for ACT imaging: ACT imaging ultimately occurs at the spectrometer focal plane. This implies that both the optical spot size across the entrance slit, governed by the telescope mirrors, and the optical performance of the spectrometer re-imaging system contribute to the optical spot size at the spectrometer focal plane. SPEXone acquires data in the spatial direction at superpixels of $11 \mu \mathrm{m}$ and aims at an oversampling ratio smaller, but close to 2. This means that the FWHM of the optical spot size at the spectrometer focal plane should ideally be smaller, but close to $22 \mu \mathrm{m}$, for all wavelengths and viewing angles.

3. Optical quality for spectral imaging: The spectral resolution of the spectrometer is determined by the re-imaging of the entrance slit on the focal plane. In order to achieve a uniform spectral resolution across the detector, the size of the reimaged slit should be dominated by the constant slit width and not by the (potentially more varying) spot size. Therefore, the optical spot size in the spectral direction at the spectrometer focal plane should be smaller than $27 \mu \mathrm{m}$.

\subsection{Optical performance of the telescope}

The nominal optical design of the telescope yields a diffraction limited performance at the telescope focal plane for the $0^{\circ}$ and $\pm 20^{\circ}$ viewing angles and close to diffraction limited performance performance for the $\pm 50^{\circ}$ viewing angles. The nominal spot size in both the ALT and ACT direction is smaller than $10 \mu \mathrm{m}$.

The optical performance at the focal plane has been assessed during the integration phase of the SPEXone instrument. After mounting the telescope mirrors in the telescope housing, a pick-off mirror was placed in the space where the PMO would be integrated, thereby directing the telescope focal plane out of the telescope housing. This enabled the placement of a commercial CMOS sensor at the redirected focal plane with which images of optical spots could be captured. As a source, a simple collimator was build consisting of a narrow core fiber positioned at the focus of a spherical lens. The measurements were performed at a wavelength of $515 \mathrm{~nm}$.

The measured spots as well as the simulated spots using the optical model are shown in Fig. 2. Particularly for the $\pm 20^{\circ}$ and $\pm 50^{\circ}$ viewing angles, nice circular spots are observed, all with FWHM of $10 \mu \mathrm{m}$ in both dimensions, in perfect agreement with the simulated spots. Only for the $0^{\circ}$ viewing angle, the spots shape is elliptical, with a FWHM of $16 \mu \mathrm{m}$ in the ALT direction. This is observed at multiple positions within the field-of-view of the telescope.

Since all ALT spot sizes are well below the slit width and since all ACT spot sizes are very close to the nominal, simulated spots sizes, the telescope performance is excellent and according to the requirements. 


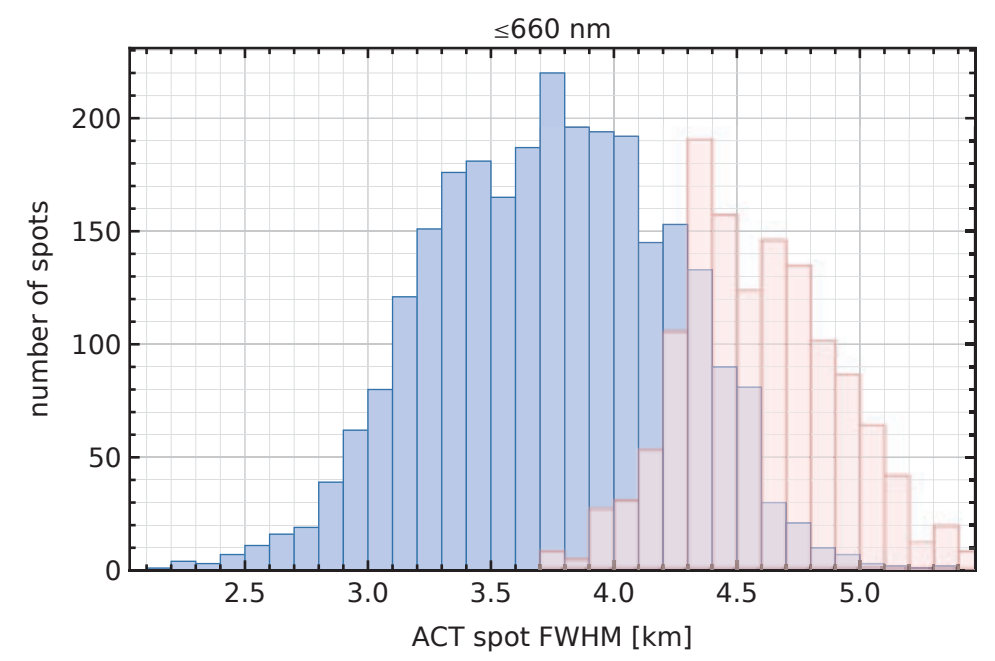

Figure 3. Spot size distribution at the spectrometer focal plane. Blue represent measured data, pink represents simulated data after tolerance analysis of the optical design.

\subsection{Optical performance of the spectrometer}

Optimizing an optical system to a finite, uniform spot size is a very challenging task, since it is generally easier to optimize an optical system to have an optical quality better than a certain threshold. A full tolerance analysis has been performed on the optical design to assess the spot size in ACT, which includes misalignments of optical components in position and orientation, manufacturing tolerances on surface shape including slope errors, and thermo-mechanical distortions. This analysis shows that, starting with a nominal design with all ACT spot sizes smaller than $11 \mu \mathrm{m}$, the expected ACT spot size distribution in the spectrometer focal plane has a mean of 18.7 $\mu \mathrm{m}$ and FWHM of $3.3 \mu \mathrm{m}$. This corresponds to a mean of $4.6 \mathrm{~km}$ and a FWHM of $0.8 \mathrm{~km}$ on ground, yielding an oversampling ratio between 1.6 and 1.9. The histogram of the ACT spot sizes averaged in ALT are shown in Fig. 3 in pink.

Spot size measurements of the integrated instrument were taken during the on-ground calibration campaign using a star stimulus (collimator) fed with diode lasers (405, 457, 515, 561, 660, 732 and 785 nm) while raster scanning the instrument across the ALT and ACT field-of-view of each viewing angle. The ACT spot sizes are extracted from 2D fits of the measured point spread functions, translated to $\mathrm{km}$ on ground, and presented as a histogram in Fig. 3 in blue. Clearly, the optical performance is better than results from the tolerance analysis. Limited star stimulus measurements taken as part of the detector alignment during instrument AIT already pointed in this direction. However, it was decided not to deliberately defocus the system, since a defocus did not result in a uniform increase of the spot size and would increase the sensitivity e.g. to settling effects during vibration qualification and gravity release effects. The measured ACT spot size distribution has an effective mean of $3.6 \mathrm{~km}(14.7 \mu \mathrm{m})$ and a FWHM of $1.3 \mathrm{~km}(5.3 \mu \mathrm{m})$, yielding an average optical oversampling ratio of 1.4. We expect this is still sufficient for adequate resampling of the data from all viewing angles to a common spatial grid.

Not taken into account in the oversampling assessment is a particular feature of this spectrometer, the socalled slit-keystone. ${ }^{16}$ Slit keystone manifests itself such that points across the slit, in the ALT-direction, are not parallel to the local keystone of the spectrometer, see Fig. 4-top. Slit keystone results in spatial spectral mixing of pixels with different ACT when extracting a spectrum from a single ground pixel from a detector image. Due to this mixing, effectively the ACT oversampling will increase as the effective optical quality across the slit is degraded.

The slit keystone of the as-designed instrument is largest near the edges of the focal plane and increases up to $6.4 \mu \mathrm{m}$ at the blue end of the spectral range. The actual keystone of the as-build instrument can be assessed 

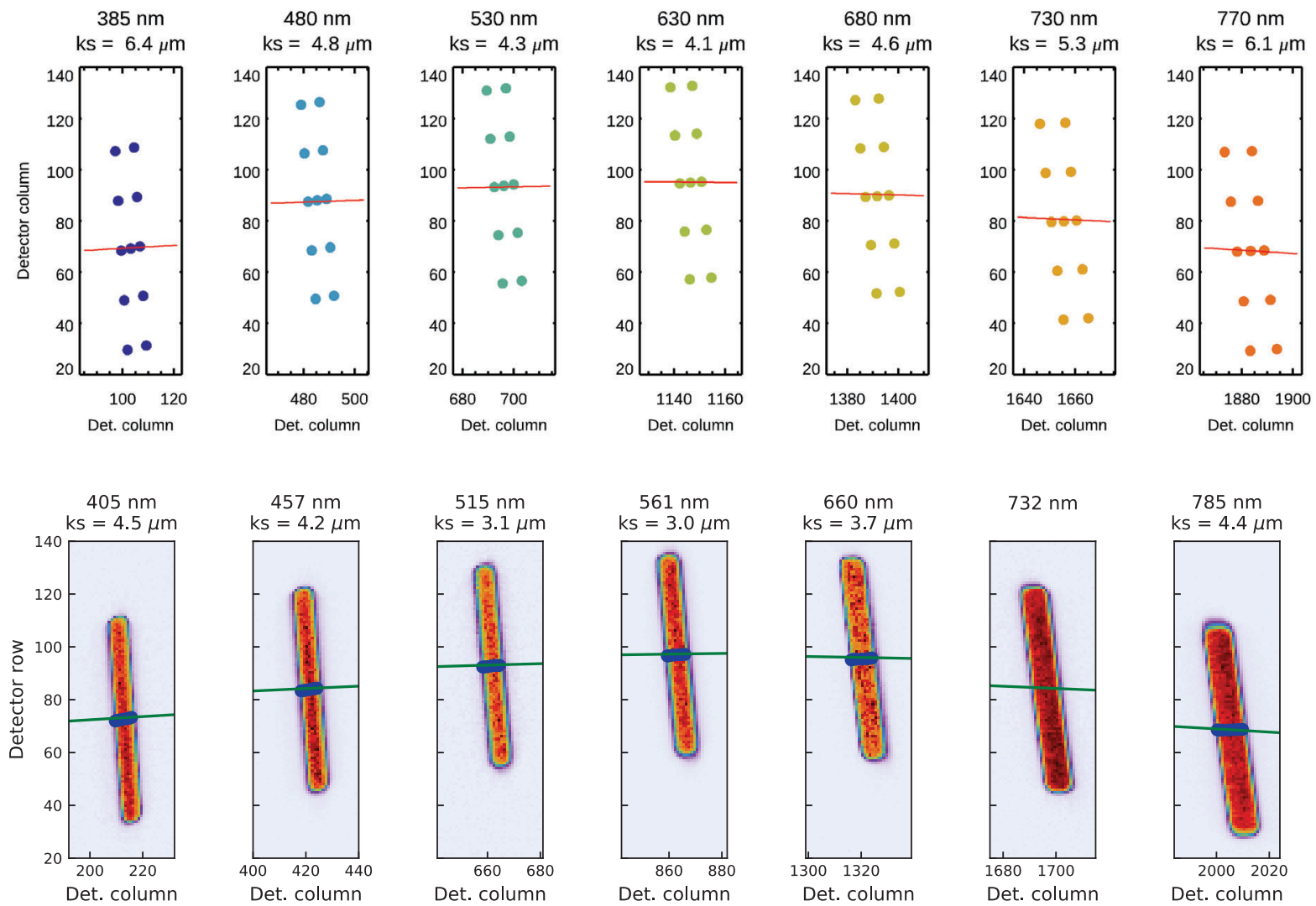

Figure 4. Slit keystone in $\mu \mathrm{m}$ for slit images of the $-50^{\circ}$ viewing angle at 7 different wavelengths. Horizontal (ALT) and vertical (ACT) dimensions are in detector pixels. Top: as-designed projections of 11 field-angles across the slit. The red line indicates the trace of a spectrum from a single ground pixel, indicating the (global) keystone in the spectrometer. Bottom: slit images obtained with wide field illumination using laser diodes. Blue dots indicate the positions of laser spots for varying ALT positions at the ACT-center of the slit. The green line represents the spectral trace extracted from white light illumination with a collimator.

using the raster-scan data obtained with diode laser illumination (as above), and with a white light illumination. The former yields the positions of points at the same ACT coordinate across the slit, while the latter yields the full spectral trace of (white) light originating from a single ground pixel. Fig. 4-bottom shows the individual slit images measured under wide-field illumination of the instrument by an integrating sphere that is fiber-fed with the 7 diode lasers. Overplotted are the individual positions of the laser spots at ACT-center in blue, and spectral trace obtained from the white light measurement in green, similar to Fig. 4-top. The slit keystone is obtained by subtracting the slope of the laser positions and the spectral trace. As can be seen, the observed slit keystone follows similar variation with wavelengths as the slit keystone in the design, but is overall roughly $25 \%$ smaller. Since the measured slit keystone is at maximum less than one-third of the mean FWHM spot size, the impact on the ACT oversampling is limited to less than $5 \%$.

\subsection{Stray light}

Stray light is the dominant contributor to the absolute absolute radiometric and systematic polarimetric error in non-uniform scenes, see. ${ }^{15}$ Full slit stray light analysis results from ZEMAX were obtained for all the view ports and various wavelengths. A representative simulated image (viewing angle $+50^{\circ}, 490 \mathrm{~nm}$ ) is displayed in Fig. 5-left, in which three main contributors with different signatures can be identified: 

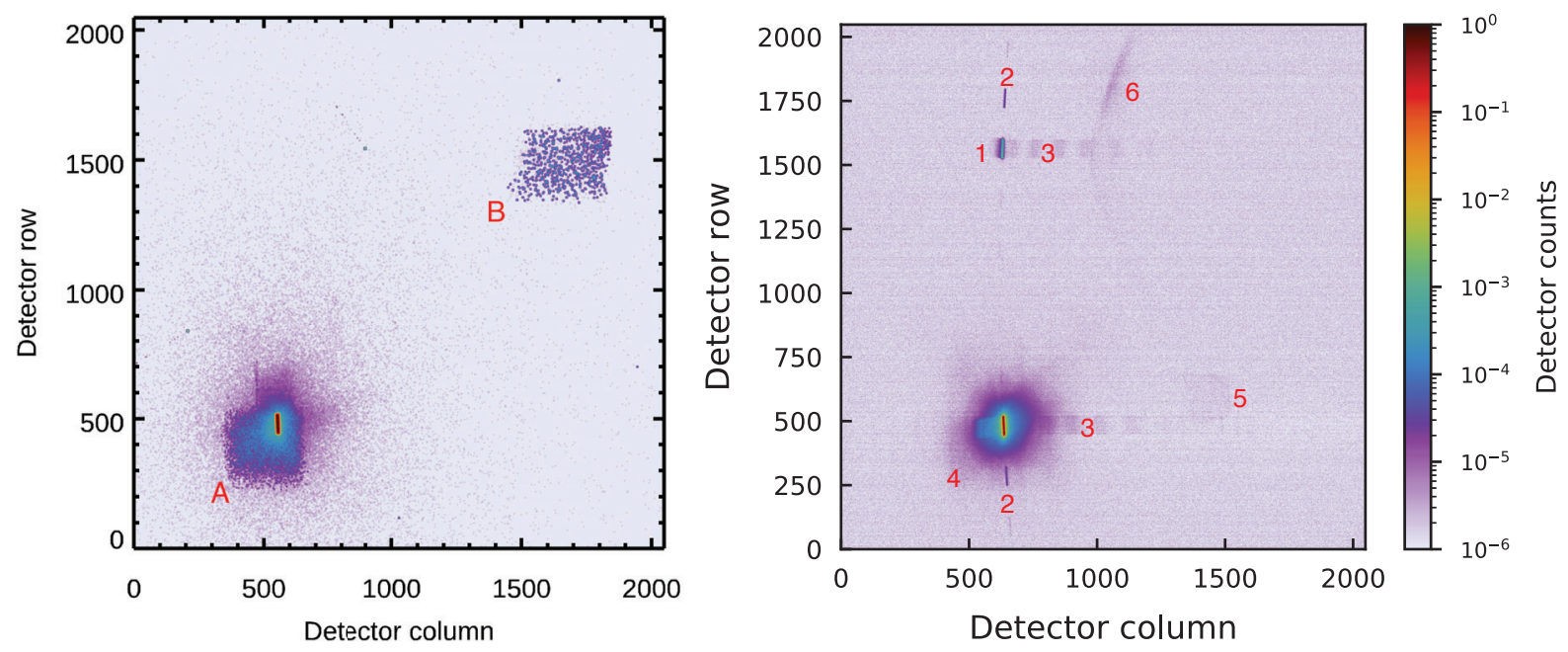

Figure 5. Simulated (left) and measured (right) stray light images for viewing angle $+50^{\circ}$ at $490 \mathrm{~nm}$ and $515 \mathrm{~nm}$. Particular features are labeled for further reference in the main text. Detector counts are relative to the maximum signal in the unsaturated image.

1. Diffuse stray light due to scattering from all optical components with finite surface roughness and of the housing and mounting structures results in a weak halo of increased intensity around the nominal optical spot and extends over relatively large distances. The total amount of diffuse stray light is around $0.7 \%$ of the nominal intensity.

2. Ghosts surrounding the (full slit) signal whose relative position depends on wavelength and viewing angle (A). These ghost reflections originate in two locations, in the PMO and in the DEM. In the PMO, 10 parallel surfaces give rise to 45 first order reflections, giving rise to an average relative ghost intensity is $0.2 \%$, whereas the ghost relative power peaks at $400 \mathrm{~nm}(0.6 \%)$ and $770 \mathrm{~nm}(0.45 \%)$. Ghost reflections in the DEM originate from reflections between the detector surface, the detector window and the order-sorting filter, giving rise to total first order reflections of about $0.8 \%$.

3. A weak ghost that overlaps in the complementary polarized image with the spectral image of the same viewing angle, but with a shifted spectral position (B). This ghost originates from light reflected at one of the DEM optical surfaces that has travelled all the way back to one of the collimator mirrors, and to the PMO, and has reflected back towards the detector from the backside of multiple PMO components. Importantly, this particular reflections can give rise to cross-talk between the two polarization channels.

Actual stray light measurements with full slit illumination have been taken during the on-ground calibration of SPEXone. Diode lasers were fed to an integrating sphere and each viewing angle was pointed subsequently to the center of the sphere. A polarizer in front of the illuminated viewing angle was set to an orientation such that the angle of polarization maximises the signal ratio between the two complementary spectral images for the diode laser wavelength. Acquisitions at three exposure times were taken in order to increase the dynamic range. The smallest exposure time of $60 \mathrm{~ms}-150 \mathrm{~ms}$ was set such that the maximum signal for a particular laser wavelength was about half the full well capacity, The longest exposure time was always $6000 \mathrm{~ms}$ and the intermediate exposure time was set to $600-1000 \mathrm{~ms}$ in order to have approximately equal steps in dynamic range. An example of a superimage, constructed using the three exposures, is shown in Fig. 5-right. Clearly, some striking differences are observed between simulated image and measured superframe.

Firstly, a nonzero signal in the complementary spectral image (1) can be observed, since the dynamic range of the superframe is larger than the extinction ratio of the polarizer. Secondly, setup stray light entering other viewing angles can be seen (2), as well as a weak periodic signal is seen in both spectral images (3). These 


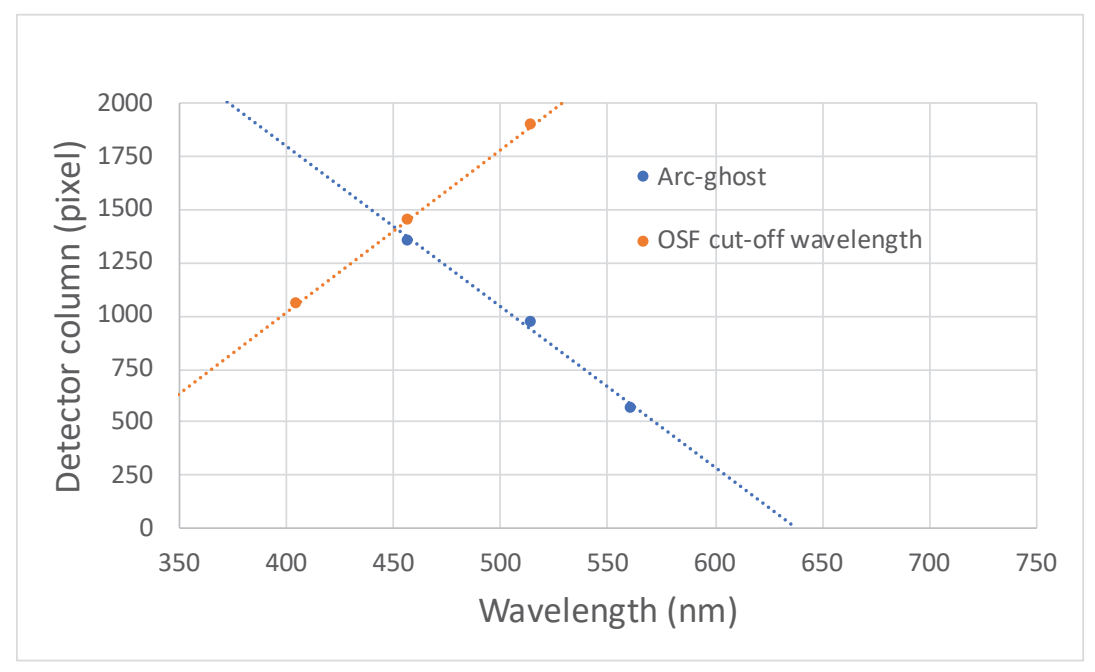

Figure 6. Position of the arc-shaped ghost column position on the focal plane versus laser wavelength. Also shown is the position of the cut-off wavelength of the order-sorting filter projected onto the detector.

result from weak, continuum illumination from the diode lasers. Due to the polarizer, this light is fully polarized resulting in the spectrally modulated intensity signal. Naturally, these signals are absent in the simulated image.

The actual measured instrument stray light shows several features, some of which are different from the simulation:

1. A halo of diffuse stray light due to scattering is visible, with somewhat higher signal near the spectral image and a 'wing' of increased signal in the spectral direction towards lower wavelengths.

2. Ghost images surrounding the slit image are very weak in comparison with the simulated image. The extended moving ghost seen in the simulation (A) cannot be well identified in the measurements at location (4).

3. A weak ghost in the same channel as the main slit image (5), which is shifted in spectral position. This ghost seems similar to ghost B in the simulation, with only the position mirrored with respect to the center detector row.

4. A weak arc-shaped ghost (6) at the position of the complimentary slit image, but shifted in wavelength. Most notably, this ghost is observed at several wavelengths, but not all, and the spectral position moves opposite with respect to the position of main slit image. At $457 \mathrm{~nm}$, this ghost is very weak, and at $405 \mathrm{~nm}$ and at $660 \mathrm{~nm}$ and higher wavelengths, this ghost is absent. This particular ghost could not be identified in the simulation and requires further investigation. Especially the opposite movement with wavelength is not observed for the main ghost that can be identified in the simulation.

Further investigation of the position of the arc-shaped ghost at different wavelengths has yielded the results as shown in Fig. 6. Also included is the position of the cut-off wavelength of the order-sorting filter. This figure explains why the ghost is weak at $457 \mathrm{~nm}$ and absent at lower wavelengths, because it is blocked by the order-sorting filter. Also, it explains why the ghost is not observed at $660 \mathrm{~nm}$ and higher wavelength, since its projected position falls outside of the detector area.

Overall, these results indicate that in the realized instrument, the contribution from diffuse stray light is slightly higher than in the simulations, whereas the ghost intensity is significantly lower than in the simulations, both in the region near the slit image, and further away. Since diffuse stray light is in general easier to correct than moving ghosts, this would be beneficial when employing a stray light correction algorithm. E.g the TROPOMI 
calibration team has demonstrated that stray light correction factors up to $10 \mathrm{x}$ can be achieved, ${ }^{17}$ which, when realized for SPEXone, would enable meeting the polarimetric requirement even at the most challenging nonuniform scenes. ${ }^{15}$

However, narrow-field stray light measurements show a structure in the diffuse stray light pattern that grows with wavelength, although in a fairly constant manner. These narrow-field stray light measurements, which are outside the scope of this paper, are performed using a star stimulus. The measurements provide the data from which the stray light kernels will be derived that are input to the stray light correction algorithm. Further analysis is required for assessing how accurate the diffuse stray light kernels can be determined and modelled for all wavelengths, and what stray light correction factor can be obtained. Also, the origin of the arc-shaped ghost and its impact on polarimetric accuracy (when uncorrected) will need to be assessed.

\section{CONCLUSIONS}

In this paper we have presented several aspects of the optical performance of SPEXone and compared the results with the as-designed values and simulations. The optical quality, in terms of RMS spot FWHM, at both the telescope and spectrometer focal plane are better than was anticipated based on an extensive tolerance analysis. Also the so-called slit keystone, a particular feature of this freeform optics based spectrometer, is about $25 \%$ smaller than the as-designed values. This means that the spatial and spectral resolution are well within the requirements, but that the optical oversampling ratio in the across-track direction is on average 1.4 , about $20 \%$ lower than anticipated. However, we expect this is still sufficient for adequate resampling of the data from all viewing angles to a common spatial grid. Instrument stray light measurements indicate a slightly higher contribution from diffuse stray light compared to simulations, but a significantly lower contribution of ghost. A quantitative analysis of the data, including the incorporation of derived stray light kernels in a stray light correction algorithm, will ultimately show the instrument (polarimetric) performance at the most challenging non-uniform scenes.

\section{ACKNOWLEDGMENTS}

We thank Paul Tol, Ryan Cooney, Pierre Piron, Thomas Bouchan, Alexander Eigenraam and Richard van Hees for their contribution to obtaining the results from the on-ground calibration of SPEXone presented in this paper.

SPEXone is a public-private initiative of SRON Netherlands Institute for Space Research and Airbus Defence and Space Netherlands, supported by opto-mechanical expertise from TNO. We acknowledge funding by the Ministry of Education Culture and Science (OCW), the Netherlands Space Office (NSO), and the Netherlands Organization of Scientific Research (NWO). We acknowledge NASA for hosting SPEXone on their PACE observatory as a partnered payload.

We also acknowledge our main suppliers for their contribution to realizing SPEXone; TNO, LT Ultra and NOVA ASTRON (mirror manufacturing), Optics Balzers Jena GmbH and Delta Optics (coating development), Horiba (grating), Lucassen and Kusters (precision mechanics), 3Dplus (camera module) and Hyperion Technologies (ICU).

\section{REFERENCES}

[1] van Amerongen, A., Rietjens, J., Campo, J., Dogan, E., Dingjan, J., Nalla, R., Caron, J., and Hasekamp, O., "SPEXone: a compact multi-angle polarimeter," in [International Conference on Space Optics - ICSO 2018], Sodnik, Z., Karafolas, N., and Cugny, B., eds., 11180, 223 - 236, International Society for Optics and Photonics, SPIE (2019).

[2] Werdell, P. J., Behrenfeld, M. J., Bontempi, P. S., Boss, E., Cairns, B., Davis, G. T., Franz, B. A., Gliese, U. B., Gorman, E. T., Hasekamp, O., Knobelspiesse, K. D., Mannino, A., Martins, J. V., McClain, C. R., Meister, G., and Remer, L. A., "The plankton, aerosol, cloud, ocean ecosystem (pace) mission: Status, science, advances," Bulletin of the American Meteorological Society 0 (Apr 2019).

[3] Martins, J. V., andChad Fish, T. N., Sparr, L., Fernandez-Borda, R., Schoeberl, M., and Remer, L., "Harp cubesat-an innovative hyperangular imaging polarimeter for earth science applications," Small Sat Pre-Conference Workshop, Logan Utah (Jul 2014). 
[4] Snik, F., Rietjens, J. H. H., Apituley, A., Volten, H., Mijling, B., Di Noia, A., Heikamp, S., Heinsbroek, R. C., Hasekamp, O. P., Smit, J. M., Vonk, J., Stam, D. M., van Harten, G., de Boer, J., Keller, C. U., and iSPEX citizen scientists, ., "Mapping atmospheric aerosols with a citizen science network of smartphone spectropolarimeters," Geophysical Research Letters 41, 7351-7358 (October 2014). 2014GL061462.

[5] van Harten, G., de Boer, J., Rietjens, J. H. H., Di Noia, A., Snik, F., Volten, H., Smit, J. M., Hasekamp, O. P., Henzing, J. S., and Keller, C. U., "Atmospheric aerosol characterization with a ground-based spex spectropolarimetric instrument," Atmospheric Measurement Techniques 7(12), 4341-4351 (2014).

[6] Smit, J. M., Rietjens, J. H. H., van Harten, G., Di Noia, A., Laauwen, W., Rheingans, B. E., Diner, D. J., Cairns, B., Wasilewski, A., Knobelspiesse, K. D., and et al., "Spex airborne spectropolarimeter calibration and performance," Applied Optics 58, 5695 (Jul 2019).

[7] Smit, J. M., Rietjens, J. H. H., di Noia, A., Hasekamp, O. P., Laauwen, W., Cairns, B., van Diedenhoven, B., and Wasilewski, A., "In-flight validation of SPEX airborne spectro-polarimeter onboard NASA's research aircraft ER-2," in [International Conference on Space Optics - ICSO 2018], Sodnik, Z., Karafolas, N., and Cugny, B., eds., 11180, 251 - 266, International Society for Optics and Photonics, SPIE (2019).

[8] Di Noia, A., Hasekamp, O. P., van Harten, G., Rietjens, J. H. H., Smit, J. M., Snik, F., Henzing, J. S., de Boer, J., Keller, C. U., and Volten, H., "Use of neural networks in ground-based aerosol retrievals from multi-angle spectropolarimetric observations," Atmospheric Measurement Techniques 8(1), 281-299 (2015).

[9] Hasekamp, O. P., Fu, G., Rusli, S. P., Wu, L., Noia, A. D., aan de Brugh, J., Landgraf, J., Smit, J. M., Rietjens, J., and van Amerongen, A., "Aerosol measurements by spexone on the nasa pace mission: expected retrieval capabilities," Journal of Quantitative Spectroscopy and Radiative Transfer 227, 170 - 184 (2019).

[10] Mishchenko, M. I., Cairns, B., Hansen, J. E., Travis, L. D., Burg, R., Kaufman, Y. J., Vanderlei Martins, J., and Shettle, E. P., "Monitoring of aerosol forcing of climate from space: analysis of measurement requirements," Journal of Quantitative Spectroscopy and Radiative Transfer 88, 149-161 (Sep 2004).

[11] Hasekamp, O. P. and Landgraf, J., "Retrieval of aerosol properties over land surfaces: capabilities of multiple-viewing-angle intensity and polarization measurements," Appl. Opt. 46, 3332-3344 (Jun 2007).

[12] Snik, F., Karalidi, T., and Keller, C. U., "Spectral modulation for full linear polarimetry," Appl. Opt. 48, 1337-1346 (Mar 2009).

[13] Campo, J., Tol, P., Smit, M., van der Vlugt, J., Talsma, J., Johansen, J., Eigenraam, A., van Hees, R., Hasekamp, O., Oort, M., van Amerongen, A., and Rietjens, J., "Characterization and video chain development of the CMOS detector applied in the multi-angle spectro-polarimeter SPEXone," in [International Conference on Space Optics - ICSO 2020], International Society for Optics and Photonics, SPIE (2021).

[14] van Brakel, R., van Amerongen, A., Rietjens, J., Oort, M., and Doornink, J., "Spexone polarimeter instrument thermal design," International Conference on Environmental Systems (Jul 2019).

[15] Rietjens, J., Campo, J., Chanumolu, A., Smit, M., Nalla, R., Fernandez, C., Dingjan, J., van Amerongen, A., and Hasekamp, O., "Expected performance and error analysis for SPEXone, a multi-angle channeled spectropolarimeter for the NASA PACE mission," in [Polarization Science and Remote Sensing IX], Craven, J. M., Shaw, J. A., and Snik, F., eds., 11132, 34 - 47, International Society for Optics and Photonics, SPIE (2019).

[16] Caron, J. and Bäumer, S., "Progress in freeform mirror design for space applications," in [International Conference on Space Optics - ICSO 2020], International Society for Optics and Photonics, SPIE (2021).

[17] Tol, P. J. J., van Kempen, T. A., van Hees, R. M., Krijger, M., Cadot, S., Snel, R., Persijn, S. T., Aben, I., and Hoogeveen, R. W. M., "Characterization and correction of stray light in tropomi-swir," Atmospheric Measurement Techniques 11(7), 4493-4507 (2018). 